\title{
AUC-Dependent Cytotoxicity of Cyclophosphamide against Human Tumors Transplanted into Nude Mice
}

\author{
Masatoshi Sugiyama, ${ }^{1, *}$ Kentarou OKamura, ${ }^{1}$ \\ Mitsuyoshi Goto, ${ }^{2}, * *$ and Morihisa $\mathrm{KITANO}^{3}$ \\ ${ }^{1}$ Department of Pharmacy, Fukui Medical School Hospital, \\ Matsuoka-cho, Fukui 910-11, Japan \\ ${ }^{2}$ Department of Pharmacy and ${ }^{3}$ Department of Thoracic Surgery, \\ Tenri Hospital, Tenri 632, Japan
}

(Received March 6, 1986)

\begin{abstract}
Summary We studied the cytotoxicity of cyclophosphamide following the administration of phenobarbital or chloral hydrate to nude mice bearing human tumor xenografts. Cyclophosphamide, $60 \mathrm{mg} / \mathrm{kg}$, was injected intraperitoneally once a week for four weeks. The antitumor efficacy of cyclophosphamide was not altered by pretreatment with phenobarbital, but was significantly increased by pretreatment with chloral hydrate. In a parallel study, we measured the concentration of blood NBP-alkylating metabolites in nude mice after administration of cyclophosphamide, $60 \mathrm{mg} / \mathrm{kg}$. The AUC (area under blood decay curve) values of NBP-alkylating metabolites were $299 \pm 39,270 \pm 13$, and $521 \pm 57 \mathrm{nmol}$ eq nor-mustard $\mathrm{ml}^{-1} \cdot \mathrm{h}$ in the controls, phenobarbital-pretreated, and chloral hydrate-pretreated groups, respectively. In contrast, $C_{\max }$ (maximal concentration) values did not show any significant differences among these three groups. An increase in the AUC value of NBP-alkylating metabolites might have led to the stimulation of cytotoxicity of cyclophosphamide in the chloral hydrate-pretreated group. These results indicate that cyclophosphamide possesses AUC-dependent cytotoxicity against human tumor.
\end{abstract}

Key Words: cyclophosphamide, phenobarbital, chloral hydrate, NBPalkylating metabolites, AUC

Cyclophosphamide, an antineoplastic agent which is classified as an alkylator, does not manifest cytotoxic activity until metabolized by the mixed function oxidases of liver microsomes [1-4]. Drugs which affect these mixed function oxidases

\footnotetext{
*To whom correspondence should be addressed.

**Present address: Department of Hospital Pharmacy, Nagoya Memorial Hospital, Nagoya 468, Japan
} 
might therefore be expected to alter the activation of cyclophosphamide. Many studies on its interaction with phenobarbital, SKF-525A, or chloral hydrate have been performed, but assessments of the effects of these drugs on cyclophosphamide cytotoxicity are divided.

In the present study, we examined the effect of phenobarbital or chloral hydrate pretreatment on cyclophosphamide cytotoxicity against human tumors transplanted into nude mice. In a parallel study, the 4-( $p$-nitro-benzyl)pyridine (NBP)alkylating metabolite concentrations in the blood were measured, and pharmacokinetic values were estimated. The NBP-alkylating metabolites which are determined by the NBP reaction are not always identified with potent cytotoxic metabolites. Of the various metabolites of cyclophosphamide, 4-hydroxycyclophosphamide is the most potent cytotoxic species [5-9]. However, the NBP-alkylating metabolites include not only 4-hydroxycyclophosphamide but also phosphoramide mustard, carboxyphosphamide, and nor-nitrogen mustard [3]. On the other hand, a positive correlation between blood NBP-alkylating metabolite concentrations and cytotoxic activity of cyclophosphamide has been shown in a study using Walker 256 sarcoma [10]. This paper describes the relationship between cytotoxicity of cyclophosphamide and blood NBP-alkylating metabolite concentrations in nude mice.

\section{MATERIALS AND METHODS}

Mice. Eight- to ten-week-old male BALB/c Jcl-nu mice (nude mice, Clea Japan, Inc., Tokyo) weighing 20 to $25 \mathrm{~g}$ were used. Nude mice were housed in a laminar air-flow room under aseptic conditions. Sterilized bedding, cages, food, and water were used.

Human tumor. The tumor obtained by surgery was a metastatic lung cancer from osteosarcoma and was serially transplanted in nude mice. In this study, eighth-generation cells were used. This tumor was shown to possess high sensitivity to cyclophosphamide in a previous experiment using sixth-generation cells.

Chemotherapeutic agents. Cyclophosphamide (Endoxan ${ }^{\circledR}$, Shionogi \& Co., Ltd., Osaka) and phenobarbital (Phenobal ${ }^{\circledR}$, Sankyo Co., Ltd., Tokyo) were used in parenteral form. Chloral hydrate (Nakarai Chemicals, Ltd., Kyoto) in crystalline form was dissolved in sterile distilled water and filtered through a $0.22-\mu \mathrm{m}$ pore-size filter (Terumo Co., Tokyo).

Examination of cyclophosphamide cytotoxicity. The tumor was removed from nude mice aseptically and minced into pieces about $2 \times 2 \times 2 \mathrm{~mm}$ in size. These minced fragments were transplanted subcutaneously with a trocar into the backs of nude mice. The animals were divided into six groups as follows: controls; phenobarbital only; chloral hydrate only; cyclophosphamide only; cyclophosphamide pretreated with phenobarbital; cyclophosphamide pretreated with chloral hydrate. Treatments of cyclophosphamide were started on day 18 after tumor transplantation. Cyclophosphamide at a dose of $60 \mathrm{mg} / \mathrm{kg}$ was injected intraperitoneally 
once a week for four weeks. Phenobarbital at a dose of $80 \mathrm{mg} / \mathrm{kg}$ was injected intraperitoneally 48 and $24 \mathrm{~h}$ before cyclophosphamide injection, and chloral hydrate at a dose of $20 \mathrm{mg} / \mathrm{kg}$ was injected intraperitoneally $10 \mathrm{~min}$ before cyclophosphamide injection. Cytotoxic effects were partially evaluated according to the Battelle Columbus Laboratories method [11] as follows: The tumor weight was calculated using the formula: tumor weight $(\mathrm{mg})=W^{2} \times L / 2$, where $W$ and $L$ are the lengths in $\mathrm{mm}$ of the short and long axes, respectively. Relative mean tumor weight $(R W)$ was estimated by the formula: $R W=W_{\mathrm{i}} / W_{\mathrm{o}}$, where $W_{\mathrm{i}}$ is the mean tumor weight of a group at any time and $W_{\mathrm{o}}$ is the mean tumor weight of that group at the initiation of treatment. Statistical significances of differences were determined between the $R W$ values for the groups.

Determination of NBP-alkylating metabolites and analysis of blood concentrations. Cyclophosphamide, phenobarbital, or chloral hydrate was administered at the same doses as those used in the examination of cytotoxicity. Blood samples were drawn from the infraorbital veniplex at $0.1,0.25,0.5,1,1.5$, and $2 \mathrm{~h}$ after intraperitoneal injection of cyclophosphamide and assayed by a modification of the method of Friedman and Boger [12] and Morita et al. [13]. One-tenth-milliliter samples of blood were each diluted to $1.5 \mathrm{ml}$ with iced distilled water. A $1.5-\mathrm{ml}$ volume of $4 \%$ perchloric acid was added to the $1.5 \mathrm{ml}$ diluted sample and centrifuged at $3,000 \mathrm{rpm}$ for $10 \mathrm{~min}$ at $0^{\circ} \mathrm{C}$. A 2-ml volume of supernatant was transferred to another tube, an appropriate volume of mixture solution $(0.2 \mathrm{M}$ acetate buffer, $\mathrm{pH} 4.6: 1 \mathrm{~N} \mathrm{NaOH}=7: 3$ ) was added, and the $\mathrm{pH}$ was adjusted to 4.6. One-half of the final volume of $5 \%$ NBP in acetone was added and heated at $100^{\circ} \mathrm{C}$ for $20 \mathrm{~min}$. After cooling, $2 \mathrm{ml}$ of acetone, $1 \mathrm{ml}$ of ethylene dichloride, and $2 \mathrm{ml}$ of $1 \mathrm{~N} \mathrm{NaOH}$ were added consecutively and shaken for $10 \mathrm{~s}$. This solution was placed on ice for $25 \mathrm{~min}$ and then centrifuged at 3,000 rpm for $5 \mathrm{~min}$. The upper layer was discarded and the lower layer was used for determination with a spectrophotometer (Model 220A, Hitachi, Ltd., Tokyo) at $540 \mathrm{~nm}$.

The pharmacokinetic values of NBP-alkylating metabolites in the blood were estimated according to the following formula reported by Brock et al. [14]:

$$
y=\frac{k_{1}}{k_{1}-k_{2}} \cdot y_{0}\left(e^{-k_{2} t}-e^{-k_{1} t}\right)
$$

$y$, concentration of NBP-alkylating metabolites in the blood; $y_{0}$, initial concentration of NBP-alkylating metabolites in the liver; $k_{1}$, rate at which NBP-alkylating metabolites pass from the liver into the blood; $k_{2}$, rate at which NBP-alkylating metabolites are eliminated from the blood.

$$
\begin{aligned}
\mathrm{AUC} & =\frac{k_{1}}{k_{1}-k_{2}} \cdot y_{0} \int_{0}^{\infty}\left(e^{-k_{2} t}-e^{-k_{1} t}\right) \mathrm{d} t \\
C_{\mathrm{max}} & =\frac{k_{1}}{k_{1}-k_{2}} \cdot y_{0}\left(e^{-k_{2} t p}-e^{-k_{1} t p}\right) \\
t p & =\frac{1}{k_{1}-k_{2}} \cdot \ln \frac{k_{1}}{k_{2}}
\end{aligned}
$$

Vol. 1, No. 2, 1986 
These values were calculated using the iterative least squares computer program MULTI [15] with an N5200 model 05 computer (NEC Co., Tokyo).

Purification of liver microsomal P-450 [16]. Nude mice pretreated with phenobarbital or chloral hydrate were killed, and their livers were resected. The liver was homogenized with 4 volumes of $1.15 \% \mathrm{KCl}$ solution in a Potter glass homogenizer equipped with a Teflon pestle. The homogenate was centrifuged at 9,000 $\times g$ for $30 \mathrm{~min}$, and the precipitate was discarded. The supernatant was then centrifuged at $105,000 \times g$ for $60 \mathrm{~min}$ in an ultracentrifuge (Type 55P-72, Hitachi Koki Co., Ltd., Tokyo). The supernatant was decanted, and the pellet was dispersed in the $1.15 \% \mathrm{KCl}$ solution, again using a Potter glass homogenizer. This suspension was then centrifuged again as above. The pellet obtained from these procedures was used as the microsomal preparation, and was suspended in 12 volumes (liver weight) of $50 \mathrm{~mm}$ phosphate buffer solution, $\mathrm{pH}$ 7.4, containing $1 \mathrm{~mm}$ EDTA.

Difference spectra of microsomal suspensions were measured with a spectrophotometer in the range of 400 to $500 \mathrm{~nm}$. Microsomal suspensions were placed in both the sample and reference cells. After recording of the baseline, $\mathrm{CO}$ was bubbled through the sample suspension for $20 \mathrm{~s}$. Also, a few milligrams of solid sodium dithionite was added to both suspensions. $\mathrm{CO}$ was again bubbled through the sample suspension, and the difference spectrum was measured. The content of $\mathrm{P}-450$ was determined from the $\mathrm{CO}$ difference spectrum with a molar extinction difference of $91 \mathrm{~mm}^{-1} \cdot \mathrm{cm}^{-1}$ between 450 and $490 \mathrm{~nm}$. The protein concentration of microsomal suspension was measured by the Folin-Lowry method [17]. P-450 values were represented as the P-450 content per mg protein.

\section{RESULTS}

Cytotoxicity of cyclophosphamide against human tumors transplanted into nude mice. The antitumor effects of cyclophosphamide against human tumors transplanted into nude mice are shown in Fig. 1. Inhibitive effects on tumor growth were observed in the three groups treated with cyclophosphamide, but were not observed in the other groups.

Table 1 shows the $R W$ values in the three groups treated with cyclophosphamide. A significant difference $(p<0.05)$ was found at day-39 (fourth treatment of cyclophosphamide) between the chloral hydrate-pretreated $(0.65 \pm 0.06)$ and phenobarbital-pretreated $(0.98 \pm 0.11)$ groups. The $R W$ values at day-53, 67, and 81 were as follows: $0.55 \pm 0.05,0.54 \pm 0.06$, and $0.49 \pm 0.06$ for the chloral hydrate-pretreated group; $0.88 \pm 0.10,0.80 \pm 0.11$, and $0.95 \pm 0.22$ for the cyclophosphamide alone group; and $1.02 \pm 0.13,1.13 \pm 0.20$, and $1.20 \pm 0.22$ for the phenobarbital-pretreated group, respectively. The antitumor efficacy of cyclophosphamide against this human tumor was significantly increased by pretreatment with chloral hydrate, whereas it was not altered by pretreatment with phenobarbital.

Blood concentration of NBP-alkylating metabolites. Figure 2 shows the mean blood concentrations of NBP-alkylating metabolites in nude mice after intra- 


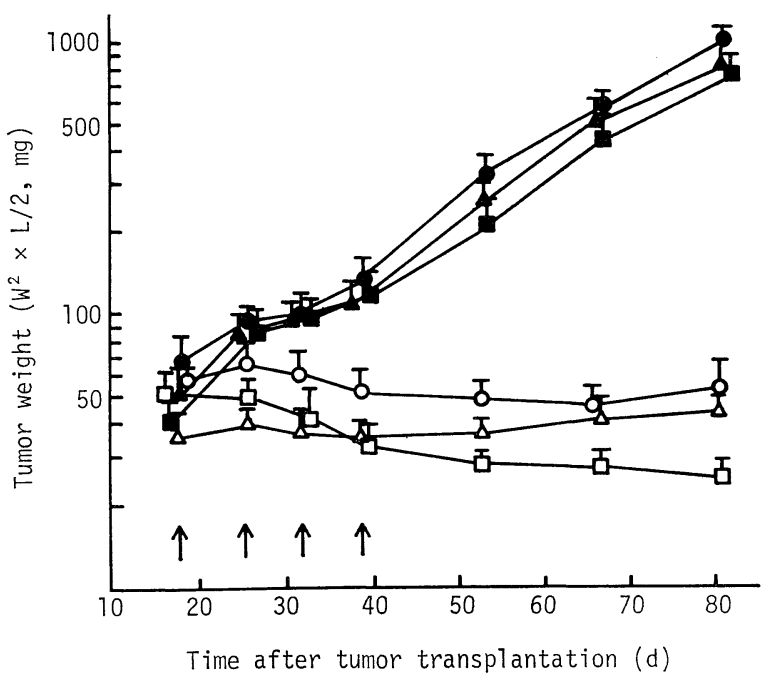

Fig. 1. Antitumor effect of cyclophosphamide against human tumors transplanted into nude mice. Arrows (18, 26, 32, and 39 d) indicate i.p. injections of cyclophosphamide, $60 \mathrm{mg} / \mathrm{kg}$. Phenobarbital, $80 \mathrm{mg} / \mathrm{kg}$, was injected i.p. 48 and $24 \mathrm{~h}$ before cyclophosphamide. Chloral hydrate, $20 \mathrm{mg} / \mathrm{kg}$, was injected i.p. $10 \mathrm{~min}$ before cyclophosphamide. Each point represents the mean $\pm \mathrm{SE}$ from at least 5 mice. $O$, Cyclophosphamide alone; $\triangle$, pretreated with phenobarbital; $\square$, pretreated with chloral hydrate; $\boldsymbol{\Delta}$, phenobarbital alone; chloral hydrate alone;

, control.

Table 1. Comparison of relative mean tumor weight $(R W)$.

\begin{tabular}{cccc}
\hline $\begin{array}{c}\text { Time after tumor } \\
\text { transplantation } \\
\text { (d) }\end{array}$ & \multicolumn{2}{c}{ Relative mean tumor weight $\left(R W=W_{\mathrm{i}} / W_{\mathrm{o}}\right)$} \\
\cline { 2 - 4 } & $\begin{array}{c}\text { Cyclophosphamide } \\
\text { alone }\end{array}$ & $\begin{array}{c}\text { Pretreated with } \\
\text { phenobarbital }\end{array}$ & $\begin{array}{c}\text { Pretreated with } \\
\text { chloral hydrate }\end{array}$ \\
\hline 18 & $1.00 \pm 0.13$ & $1.00 \pm 0.12$ & $1.00 \pm 0.18$ \\
26 & $1.14 \pm 0.20$ & $1.09 \pm 0.13$ & $0.95 \pm 0.13$ \\
32 & $1.04 \pm 0.19$ & $1.03 \pm 0.13$ & $0.81 \pm 0.09$ \\
39 & $0.89 \pm 0.16$ & $0.98 \pm 0.11$ & $0.65 \pm 0.06^{*}$ \\
53 & $0.88 \pm 0.10$ & $1.02 \pm 0.13$ & $0.55 \pm 0.05^{* *}$ \\
67 & $0.80 \pm 0.11$ & $1.13 \pm 0.20$ & $0.54 \pm 0.06^{* *}$ \\
81 & $0.95 \pm 0.22$ & $1.20 \pm 0.22$ & $0.49 \pm 0.06^{* *}$ \\
\hline
\end{tabular}

Each value represents the mean $\pm \mathrm{SE}$ from at least 5 mice. $* p<0.05$ relative to cyclophosphamide alone or pretreatment with phenobarbital; ${ }^{* *} p<0.01$ relative to pretreatment with phenobarbital; $p<0.05$ relative to cyclophosphamide alone.

peritoneal injections of cyclophosphamide at $60 \mathrm{mg} / \mathrm{kg}$. The time to reach $C_{\max }$ was reduced by pretreatment with phenobarbital, whereas a high concentration was maintained for a long period by pretreatment with chloral hydrate.

The pharmacokinetic values are listed in Table 2. AUC values for chloral hydrate-pretreated, cyclophosphamide alone, and phenobarbital-pretreated groups were $521 \pm 57,299 \pm 39$, and $270 \pm 13 \mathrm{nmol}$ eq nor-mustard $\mathrm{ml}^{-1} \cdot \mathrm{h}$, respectively. 


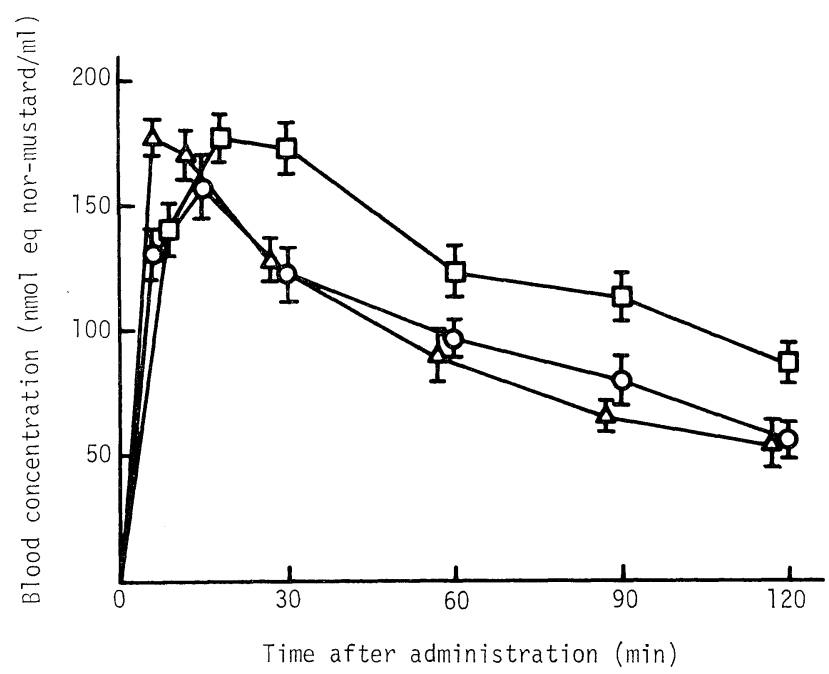

Fig. 2. Blood concentrations of NBP-alkylating metabolites in nude mice after i.p. injection of cyclophosphamide. Cyclophosphamide, $60 \mathrm{mg} / \mathrm{kg}$, was injected i.p. Phenobarbital, $80 \mathrm{mg} / \mathrm{kg}$, was injected i.p. 48 and $24 \mathrm{~h}$ before cyclophosphamide. Chloral hydrate, 20 $\mathrm{mg} / \mathrm{kg}$, was injected i.p. $10 \mathrm{~min}$ before cyclophosphamide. Each point represents the mean $\pm \mathrm{SE}$ from at least 5 mice. $\bigcirc$, Cyclophosphamide alone; $\triangle$, pretreated with phenobarbital; $\square$, pretreated with chloral hydrate.

Table 2. Pharmacokinetic values of NBP-alkylating metabolites in nude mice after i.p. injection of cyclophosphamide $(60 \mathrm{mg} / \mathrm{kg})$.

\begin{tabular}{lccc}
\hline & $\begin{array}{c}\text { Cyclophosphamide } \\
\text { alone }\end{array}$ & $\begin{array}{c}\text { Pretreated with } \\
\text { phenobarbital }\end{array}$ & $\begin{array}{c}\text { Pretreated with } \\
\text { chloral hydrate }\end{array}$ \\
\hline $\begin{array}{c}C_{\text {max }}(\text { nmol eq } \\
\text { nor-mustard ml }{ }^{-1} \text { ) }\end{array}$ & $153 \pm 13$ & $178 \pm 4$ & $177 \pm 20$ \\
$\begin{array}{c}\text { AUC (nmol eq } \\
\left.\text { nor-mustard } \mathrm{ml}^{-1} \cdot \mathrm{h}\right)\end{array}$ & $299 \pm 39$ & $270 \pm 13$ & $521 \pm 57^{*}$ \\
$T_{1 / 2}(\mathrm{~h})$ & $1.25 \pm 0.25$ & $0.98 \pm 0.08$ & $1.86 \pm 0.43^{* *}$ \\
\hline
\end{tabular}

Each value represents the mean $\pm \mathrm{SE}$ from at least 5 mice. $* p<0.01$ relative to cyclophosphamide alone or pretreatment with phenobarbital; ${ }^{* *} p<0.01$ relative to pretreatment with phenobarbital; $p<0.05$ relative to cyclophosphamide alone.

Table 3. Liver microsomal P-450 content in nude mice.

\begin{tabular}{lc}
\hline \multicolumn{1}{c}{ Treatments } & $\begin{array}{c}\text { P-450 } \\
\text { (nmol/mg protein) }\end{array}$ \\
\hline Control & 0.726 \\
Pretreated with phenobarbital & 1.497 \\
Pretreated with chloral hydrate & 0.702 \\
\hline
\end{tabular}

Phenobarbital $(80 \mathrm{mg} / \mathrm{kg})$ was injected i.p. 48 and $24 \mathrm{~h}$ before sacrifice. Chloral hydrate $(20 \mathrm{mg} / \mathrm{kg}$ ) was injected i.p. $10 \mathrm{~min}$ before sacrifice. 
AUC was significantly increased by pretreatment with chloral hydrate. Half-life $\left(T_{1 / 2}\right)$ was prolonged in the chloral hydrate-pretreated group, whereas no significant differences were shown for $C_{\max }$ values between the three groups.

Inductive effect of liver microsomal P-450. The contents of liver microsomal P-450 in nude mice are shown in Table 3. The phenomenon of P-450 induction was shown in the phenobarbital-pretreated group. Chloral hydrate had no effect on the P-450 content in liver microsomes.

\section{DISCUSSION}

The activation of cyclophosphamide is altered by many drugs which affect the mixed function oxidases of liver microsomes. Pretreatment with phenobarbital, a well-known inducer of P-450, has been shown to cause a decrease in the blood AUC of NBP-alkylating metabolites and in the antitumor efficacy of cyclophosphamide against L1210 leukemia [18, 19] or P-388 leukemia [20]. However, others have observed no change in the antitumor efficacy against Walker 256 sarcoma [21] or L1210 leukemia [22] following pretreatment with phenobarbital. In contrast, however, pretreatment with phenobarbital has been shown to cause an increase in cyclophosphamide toxicity [23]. This discrepancy of results may have been caused by differences in the experimental schedules employed. In our previous study [24] using Ehrlich LP-12 tumors transplanted into BALB/c mice, pretreatment with phenobarbital at a dose of $320 \mathrm{mg} / \mathrm{kg}$ increased the antitumor efficacy of cyclophosphamide, whereas the efficacy was decreased at a dose of $80 \mathrm{mg} / \mathrm{kg}$. Chloral hydrate inhibits aldehyde oxidase or dehydrogenase competitively and leads to prolonged metabolism of cyclophosphamide [25]. Pretreatment with chloral hydrate has been demonstrated to cause an increase in the blood AUC of NBP-alkylating metabolites and in the antitumor activity of cyclophosphamide against Lewis lung carcinoma xenografts in $\mathrm{C}_{57} \mathrm{BL}$ mice or mammary carcinoma xenografts in $(\mathrm{C} 3 \mathrm{H} \times 020) F_{1}$ mice [25].

In many previous studies, there have been differences in the dosage of cyclophosphamide used for chemotherapy and in the measurement of blood concentrations of NBP-alkylating metabolites, so that discussion of the relationship between cytotoxicity and blood concentration may be difficult.

Human tumors can easily be transplanted into athymic nude mice. The characteristics and histopathology of the original human tumors have been maintained through serial transplantation in nude mice [26-28]. Up to eight generations of transplanted tumors have been successfully produced showing a human karyotype [29]. Thus, human tumors in nude mice might provide a useful experimental model for studying the cytotoxicity of antineoplastic agents.

In this study, we examined the relationship between the cytotoxicity of cyclophosphamide and blood NBP-alkylating metabolite concentrations following pretreatment with phenobarbital or chloral hydrate in human tumor-bearing nude mice. Pretreatment with phenobarbital had little effect on the cytotoxicity of 
cyclophosphamide and on the $C_{\max }$ and AUC of NBP-alkylating metabolites in blood, although an inductive effect on liver microsomal P-450 was shown. In contrast, pretreatment with chloral hydrate significantly increased the cytotoxicity of cyclophosphamide with an increase in the AUC of NBP-alkylating metabolites in blood. Since chloral hydrate did not alter the P-450 content of liver microsomes, it might competitively inhibit aldehyde oxidase or dehydrogenase, thus possibly depressing the biotransformation rate of 4-hydroxycyclophosphamide. However, the $C_{\max }$ of NBP-alkylating metabolites was not altered by chloral hydrate pretreatment. These results indicate that cyclophosphamide possesses AUC-dependent cytotoxicity against human tumor.

\section{REFERENCES}

1. Brock, N., and Hohorst, H.-J. (1963): Uber die aktivierung von cyclophosphamide in vivo und in vitro. Arzneim.-Forsch., 13, 1021-1031.

2. Brock, N., and Hohorst, H.-J. (1967): Metabolism of cyclophosphamide. Cancer, 20, 900904.

3. Cohen, J.L., and Jao, J.Y. (1970): Enzymatic basis of cyclophosphamide activation by hepatic microsomes of the rat. J. Pharmacol. Exp. Ther., 174, 206-210.

4. Connors, T.A., Grover, P.L., and McLoughlin, A.M. (1970): Microsomal activation of cyclophosphamide in vivo. Biochem. Pharmacol., 19, 1533-1535.

5. Connors, T.A., Cox, P.J., Farmer, P.B., Foster, A.B., and Jarman, M. (1974): Some studies of the active intermediates formed in the microsomal metabolism of cyclophosphamide and isophosphamide. Biochem. Pharmacol., 23, 115-129.

6. Takamizawa, A., Matsumoto, S., Iwata, T., Katagiri, K., Tochino, Y., and Yamaguchi, K. (1973): Studies on cyclophosphamide metabolites and their related compounds. II. Preparation of an active species of cyclophosphamide and some related compounds. J. Am. Chem. Soc., 95, 985-986.

7. Brock, N. (1976): Comparative pharmacologic study in vitro and in vivo with cyclophosphamide (NSC-26271), cyclophosphamide metabolites, and plain nitrogen mustard compounds. Cancer Treat. Rep., 60, 301-308.

8. Domeyer, B.E., and Sladek, N.E. (1980): Kinetics of cyclophosphamide biotransformation in vivo. Cancer Res., 40, 174-180.

9. Powers, J.F., and Sladek, N.E. (1983): Cytotoxic activity relative to 4-hydroxycyclophosphamide and phosphoramide mustard concentrations in the plasma of cyclophosphamidetreated rats. Cancer Res., 43, 1101-1106.

10. Weaver, F.A., Torkelson, A.R., Zygmunt, W.A., and Browder, H.P. (1978): Tissue culture cytotoxicity assay for cyclophosphamide metabolites in rat body fluids. J. Pharm. Sci., 67, 1009-1012.

11. Houchens, D.P., and Ovejera, A.A. (1978): The therapy of human tumors in athymic (nude) mice, in Proceedings of the Symposium on the Use of Athymic (Nude) Mice in Cancer Research, ed. by Houchens, D.P., and Ovejera, A.A., Gustav Fisher, New York, Stuttgart, pp. 267-280.

12. Friedman, O.M., and Boger, E. (1961): Colorimetric estimation of nitrogen mustards in aqueous media. Anal. Chem., 33, 906-910.

13. Morita, M., Tochino, Y., Iwata, T., and Minesita, T. (1967): Studies on cyclophosphamide. Part II. The separative determination of cyclophosphamide and nor-mustard in biological materials. Annu. Rep. Shionogi Res. Lab., 17, 114-121. 
14. Brock, N., Gross, R., Hohorst, H.-J., Klein, H.O., and Schneider, B. (1971): Activation of cyclophosphamide in man and animals. Cancer, 27, 1512-1529.

15. Yamaoka, K., Tanigawara, Y., Nakagawa, T., and Uno, T. (1981): A pharmacokinetic analysis program (MULTI) for microcomputer. J. Pharm. Dyn., 4, 879-885.

16. Omura, T., and Sato, R. (1964): The carbon monoxide-binding pigment of liver microsomes. J. Biol. Chem., 239, 2370-2378.

17. Lowry, O.H., Rosebrough, N.J., Farr, A.L., and Randall, R.J. (1951): Protein measurement with Folin phenol reagent. J. Biol. Chem., 193, 265-275.

18. Field, R.B., Gang, M., Kline, I., Venditti, J.M., and Waravdekar, V.S. (1972): The effect of phenobarbital or 2-diethylaminoethyl-2,2-diphenylvalerate on the activation of cyclophosphamide in vivo. J. Pharmacol. Exp. Ther., 180, 475-483.

19. Donelli, M.G., Vecchi, A., Bossi, A., Colombo, T., Sironi, M., Pantarotto, C., Garattini, S., and Spreafico, F. (1977): Effect of phenobarbital on cyclophosphamide cytotoxic activity and pharmacokinetics in mice. Tumori, 63, 137-146.

20. Alberts, D.S., and Van Daalen Wetters, T. (1976): The effect of phenobarbital on cyclophosphamide antitumor activity. Cancer Res., 36, 2785-2789.

21. Sladek, N.E. (1972): Therapeutic efficacy of cyclophosphamide as a function of its metabolism. Cancer Res., 32, 535-542.

22. Hart, L.G., and Adamson, R.H. (1969): Effect of microsomal enzyme modifiers on toxicity and therapeutic activity of cyclophosphamide in mice. Arch. Int. Pharmacodyn. Ther., 180, $391-401$.

23. Bus, J.S., Short, R.D., and Gibson, J.E. (1973): Effect of phenobarbital and SKF 525A on the toxicity, elimination and metabolism of cyclophosphamide in newborn mice. J. Pharmacol. Exp. Ther., 184, 749-756.

24. Sugiyama, M., Goto, M., Nakajima, S., and Yamashina, H. (1981): The effect of phenobarbital or chloral hydrate on the activation and antitumor activity of cyclophosphamide. Gan To Kagakuryoho, 8, 1968-1973.

25. Donelli, M.G., Bartosek, I., Guaitani, A., Martini, A., Colombo, T., Pacciarini, M.A., and Modica, R. (1976): Importance of pharmacokinetics studies on cyclophosphamide (NSC26271) in understanding its cytotoxic effect. Cancer Treat. Rep., 60, 395-401.

26. Povlsen, C.O., and Jacobsen, G.K. (1975): Chemotherapy of a human malignant melanoma transplanted in the nude mouse. Cancer Res., 35, 2790-2796.

27. Shimosato, Y., Kameya, T., Nagai, K., Hirohashi, S., Koide, T., Hayashi, H., and Nomura, T. (1976): Transplantation of human tumors in nude mice. J. Natl. Cancer Inst., 56, 1251-1260.

28. Giovanella, B.C., Stehlin, J.S., Williams, L.J., Lee, S.S., and Shepard, R.C. (1978): Heterotransplantation of human cancers into nude mice. A model system for human cancer chemotherapy. Cancer, 42, 2269-2281.

29. Chahinian, A.P., Beranek, J.T., Suzuki, Y., Bekesi, J.G., Wisniewski, L., Selikoff, I.J., and Holland, J.F. (1980): Transplantation of human malignant mesothelioma into nude mice. Cancer Res., 40, 181-185. 